\title{
CYTOGENETIC CHARACTERIZATION OF THE GENOME OF INTERSPECIES HYBRIDS (ALOPEX-VULPES)*
}

\author{
Monika Bugno-Poniewierska ${ }^{1 *}$, Klaudia Pawlina ${ }^{1}$, Natalia Orszulak-Wolny² ${ }^{2}$ Bartosz Woźniak ${ }^{3}$, \\ Maciej Wnuk ${ }^{3}$, Andrzej Jakubczak ${ }^{4}$, Grażyna Jeżewska-Witkowska ${ }^{4}$ \\ ${ }^{1}$ Laboratory of Genomics, National Research Institute of Animal Production, \\ 32-083 Balice n. Kraków, Poland \\ ${ }^{2}$ Interdepartmental Biotechnology Studies Program, University of Agriculture, Al. Mickiewicza 21, \\ 31-120 Kraków, Poland \\ ${ }^{3}$ Department of Genetics, Institute of Applied Biotechnology and Basic Sciences, \\ University of Rzeszow, 36-100 Kolbuszowa, Poland \\ ${ }^{4}$ Department of Biological Basis of Animal Production, University of Life Sciences in Lublin, \\ Akademicka 13, 20-950 Lublin, Poland \\ •Corresponding author: monika.bugno@izoo.krakow.pl
}

\begin{abstract}
Creation of interspecific hybrids is widely common among plants and animals in order to improve economically important traits for humans. The studied material consisted of chromosomal preparations in the metaphase stage obtained from interspecies hybrids of arctic foxes (Alopex lagopus) and red foxes (Vulpes vulpes). The aim of the study was to analyze the karyotype of the interspecific hybrids taking into account the number of chromosomes of sets $A$ and $B$. With the use of techniques of classical cytogenetics ( $\mathrm{C}$ bands, AgNOR bands) and molecular cytogenetics (FISH, PRINS) we carried out a genome analysis of Alopex-Vulpes hybrids. The results of this study showed that chromosomal markers of the interspecies hybrids are inherited from the parent species and are a result of combination of their two genomes. However, intraindividual differences are also observed which may result from aberrations of chromosome segregation during embryonic development. This may lead to the formation of different cell lines with different karyotypes (mosaicism). Moreover, chromosomes of the interspecies hybrids showed telomeric signals at the ends, in the centromers, as well as short chromosome arm rich in heterochromatin. The use of PRINS method led to identification of nucleolus organizer regions on 12 chromosomes of the interspecies hybrids. The hybridization signals obtained were characterized by different size and intensity. In addition, single copies of rDNA in the centromeric regions of several metacentric chromosomes were identified.
\end{abstract}

Key words: cytogenetics, red fox, arctic fox, hybrid (Alopex-Vulpes)

Creation of interspecific hybrids is widely common among both plants and animals. The literature review reports the creation of this type of crossbreds in order to

\footnotetext{
*The work financed from author's project of the National Science Centre, project No. N N311 542540 .
} 
improve the features of interest from the point of view of breeding such as: weight gain, better resistance to diseases, increase in productivity and efficiency (Short, 1997). Also between species of canids crossings to obtain new varieties of colors and improvement of the fur quality are often carried out. An example of these are hybrids of red foxes and arctic foxes (Alopex-Vulpes). In the 1940s Cole and Shackelford described eight kinds of hybrids taking into account the sex of input species (a feature which determines the fertility of the litter) as well as their coat color (Cole and Shackelford, 1947).

Karyotypes of both parental species differ from each other in both the diploid number of chromosomes and their morphology. The red fox karyotype is known to be $2 n=34+B$ and consists of the basic A set of 34 chromosomes and a variable number of supernumerary B chromosomes, which in most studies oscillates in the range of 0 to 8 (Beliaev et al., 1974; Gustavsson and Sundt, 1965, 1967; Świtoński, 1984; Mäkinen, 1985 b). Today, however, it is believed that the number of B chromosomes can be as high as 10 (Basheva et al., 2010). All chromosomes, except for the chromosome $\mathrm{Y}$ which is morphologically similar to the B chromosomes, are metacentric chromosomes. The karyotype of the arctic fox, unlike the one of the red fox, lacks $\mathrm{B}$ chromosomes. However, this species is characterized by a variable number of set A chromosomes: $2 n=48,2 n=49$ or $2 n=50$. This is due to Robertsonian translocations often occurring in this species, which involve a fusion of acrocentric chromosomes in centromeric regions (Świtoński, 1981). This results in a formation of metacentric chromosomes, and the number of chromosomes is reduced to $2 n=49$ or 2n = 48 (Mäkinen, 1985 a; Świtoński, 1985).

Average occurrence of particular karyotypes is not exactly known. It should be noted, however, that female foxes with $2 n=48$ or $2 n=50$ karyotype are more prolific than those having the diploid number of chromosomes of $2 \mathrm{n}=49$ (Świtoński, 1985; Mäkinen, 1985 a). All of the autosomal chromosomes, except for two pairs (subjected to Robertsonian translocations) are meta- or submetacentric chromosomes. The chromosome $\mathrm{X}$ is a medium sized chromosome. Moreover, it is the smallest of submetacentric chromosomes, while the chromosome $\mathrm{Y}$ is acrocentric (Mäkinen, 1985 a; Świtoński et al., 2003).

Due to the diversity in karyotypes of parental species of the hybrids of the red fox and the arctic fox (Alopex-Vulpes), the aim of the study was the analysis of mitotic chromosomes of the hybrids using appropriate techniques of classical and molecular cytogenetics.

\section{Material and methods}

The research material consisted of peripheral blood taken after the slaughter of three male red foxes (Vulpes vulpes), five female arctic foxes (Alopex lagopus) and 17 interspecific hybrids (Alopex-Vulpes) (five males, 12 females). The cytogenetic analysis was performed on microscope preparations of metaphase chromosomes obtained after a routine culture of peripheral blood lymphocytes. 


\section{C-banding}

Slides were incubated in $0.2 \mathrm{~N} \mathrm{HCl}$ (Sigma) for $30 \mathrm{~min}$, then washed in $\mathrm{H}_{2} \mathrm{O}$ and treated with a saturated solution of $\mathrm{Ba}(\mathrm{OH})_{2}(\mathrm{Sigma})$ for $15 \mathrm{~min}$ at $50^{\circ} \mathrm{C}$. After washing in $\mathrm{H}_{2} \mathrm{O}$, slides were incubated in $2 \times \mathrm{SSC}$ at $60^{\circ} \mathrm{C}$ for $1 \mathrm{~h}$ and stained with $5 \%$ Giemsa.

\section{FISH method}

Molecular probes specific for the dog $\mathrm{X}$ and $\mathrm{Y}$ sex chromosomes and the red fox B chromosomes suspended in hybridization mix (50\% formamide, 10\% dextran sulphate, $10 \% 20 x \mathrm{xSC}, 1 \%$ Tween 20 and $29 \% \mathrm{H}_{2} \mathrm{O}$ ) were applied on the metaphase preparations. The probes were obtained with the use of manual microdissection of the dog heterosomes and the red fox B chromosomes. In brief, the preparations were denatured in $70 \%$ formamide in saline-sodium citrate buffer $(2 \times \mathrm{SSC})$ for $2.5 \mathrm{~min}$ at $70^{\circ} \mathrm{C}$. The probes were denatured at $70^{\circ} \mathrm{C}$ for $10 \mathrm{~min}$. The hybridization was carried out in $37^{\circ} \mathrm{C}$ for two days. Post-hybridization washes were as follows: three times with $50 \%$ formamide in $2 \times \mathrm{SSC}$ and three times in $2 \times \mathrm{SSC}$ at $42^{\circ} \mathrm{C}$. The $\mathrm{X}$ and $\mathrm{B}$ probes were labeled with biotin so hybridization signals were detected by the avidinFITC (fluorescein isothiocyanate) and anti-avidin system. The Y probe was labeled with $\mathrm{Cy} 3$ which does not need any additional detection. Finally, the preparations were mounted with DAPI stain in a mounting medium. Microscopic evaluation was performed under the Opton Axiophot fluorescence microscope equipped with a camera and Lucia software.

\section{Silver staining (AgNOR)}

Silver staining was performed according to Howell and Black (1980) by incubation of the slides with colloidal developer containing 50\% $\mathrm{AgNO}_{3}$ (Sigma) for 15 min. After washing in the tap water the preparations were stained in 5\% Giemsa (Merck - Sigma) for $10 \mathrm{~s}$.

\section{PRINS with oligonucleotides complementary to rRNA}

Primers specific for the mammalian 18S ribosomal RNA gene were used (Genomed, Poland):

\section{Forward: 5'CCACGCTCTCGCCAACGTTAAT3'}

\section{Reverse: 5'CGCTGTCCCACCGTCTCCTA3'}

The PRINS reaction mixture in a total volume of $25 \mu \mathrm{l}$ per slide contained: $0.2 \mathrm{mM}$ dATP, dCTP and dGTP; $0.025 \mathrm{mM}$ dTTP; 1 nmol biotin-16dUTP; $0.2 \mathrm{U}$ Ampli Taq GOLD polymerase (Invitrogen, Germany); 1x Taq polymerase buffer with $\mathrm{MgCl}_{2}$; 0.2 FM Fwd primer and 0.2 FM Rev primer, and sterile distilled water (MilliQ). The PRINS reaction mixture was applied on a slide, covered with a coverslip and sealed with fixogum to prevent evaporation. Reaction conditions were as follows: denaturation at $95^{\circ} \mathrm{C}$ for $5 \mathrm{~min}$; annealing at $57^{\circ} \mathrm{C}$ for $15 \mathrm{~min}$ and extension at $68^{\circ} \mathrm{C}$ for $15 \mathrm{~min}$. The PRINS reaction was stopped by transferring the slides to stop buffer containing $50 \mathrm{mM} \mathrm{NaCl}$ and $50 \mathrm{mM}$ EDTA $(\mathrm{pH} 8.0)$ at $58^{\circ} \mathrm{C}$ for $5 \mathrm{~min}$ and then to the same buffer $(50 \mathrm{mM} \mathrm{NaCl}$ and $50 \mathrm{mM}$ EDTA, $\mathrm{pH}$ 8.0) at room temperature for $7 \mathrm{~min}$. 


\section{PRINS with oligonucleotides complementary to telomeric DNA}

Primers specific to both forward and reverse telomeric DNA strands were used ((TTTAGGG)7 and (CCCTAA)7, respectively). The PRINS reaction mixture in a total volume of $10 \mu \mathrm{l}$ per slide contained: $0.2 \mathrm{mM}$ dATP, dCTP and dGTP; $0.025 \mathrm{mM}$ dTTP; $1 \mathrm{nmol}$ biotin-16dUTP; 0.2 U Ampli Taq GOLD polymerase (Invitrogen, Germany); 1x Taq polymerase buffer with $\mathrm{MgCl}_{2}, 0.4 \mu \mathrm{M}$ Fwd primer and $0.4 \mu \mathrm{M}$ Rev primer, and sterile distilled water (milliQ). Reaction conditions were as follows: denaturation at $94^{\circ} \mathrm{C}$ for $2 \mathrm{~min}$; annealing at $58^{\circ} \mathrm{C}$ for $30 \mathrm{~min}$. The PRINS reaction was stopped by transferring the slides to stop buffer containing $50 \mathrm{mM} \mathrm{NaCl}$ and $50 \mathrm{mM}$ EDTA $(\mathrm{pH} 8.0)$ at $58^{\circ} \mathrm{C}$ for $5 \mathrm{~min}$ and then to the same buffer $(50 \mathrm{mM}$ $\mathrm{NaCl}$ and $50 \mathrm{mM}$ EDTA, $\mathrm{pH} \mathrm{8.0)}$ at room temperature for $7 \mathrm{~min}$. Biotin Painting Kit (3 Step) with FITC (Cambio UK) were used to visualize biotin-labeled PRINS products. Chromosome staining was performed with DAPI. Photos were captured in a fluorescence microscope OlympusBX61 equipped with a DP72 CCD camera and Olympus CellF software.

\section{Results}

In females of arctic foxes there were found two forms of karyotype: $49, \mathrm{XX}$ and $50, \mathrm{XX}$. Male red foxes had $2 \mathrm{n}=34$ with a different number of $\mathrm{B}$ chromosomes - from 1 to 4 . It was also found that the number of chromosomes in the interspecies hybrids varied not only between individuals but also between the cells of one individual and ranged from 35 to 42 . The number of B chromosomes in the studied hybrids ranged from 0 to 4 and only for four individuals no B chromosome were observed. Table 1 shows karyotypes occurring in the subsequent individuals as well as their sex.

Table 1. Observed karyotypes with the inclusion of cell lines

\begin{tabular}{|c|c|c|}
\hline $\begin{array}{c}\text { No. of an } \\
\text { interspecies hybrid }\end{array}$ & Observed karyotype & Sex \\
\hline 1 & $41+1 \mathrm{~B} / 41+2 \mathrm{~B} / 42+1 \mathrm{~B} / 42+2 \mathrm{~B}$ & $\hat{o}$ \\
\hline 2 & $41+1 B / 42+1 B$ & $\hat{0}$ \\
\hline 3 & 41 & 우 \\
\hline 4 & 41 & 우 \\
\hline 5 & $40+1 \mathrm{~B} / 40+2 \mathrm{~B} / 40+3 \mathrm{~B}$ & q \\
\hline 6 & $40 / 41 / 40+1 \mathrm{~B} / 41+1 \mathrm{~B} / 41+2 \mathrm{~B} / 42+1 \mathrm{~B}$ & $\hat{0}$ \\
\hline 7 & $40+1 \mathrm{~B} / 41+1 \mathrm{~B} / 41+2 \mathrm{~B} / 42+1 \mathrm{~B}$ & q \\
\hline 8 & $41 / 41+1 B$ & q \\
\hline 9 & $40+2 \mathrm{~B} / 41+1 \mathrm{~B} / 41+2 \mathrm{~B} / 41+3 \mathrm{~B} / 41+4 \mathrm{~B} / 42+1 \mathrm{~B} / 42+3 \mathrm{~B}$ & q \\
\hline 10 & $41+1 \mathrm{~B} / 42+1 \mathrm{~B} / 42+2 \mathrm{~B}$ & $\hat{\sigma}$ \\
\hline 11 & $42 / 41+1 \mathrm{~B} / / 42+1 \mathrm{~B} / 42+2 \mathrm{~B} / 43+1 \mathrm{~B}$ & q \\
\hline 12 & $41+1 \mathrm{~B} / 42+1 \mathrm{~B}$ & $\hat{0}$ \\
\hline 13 & $41 / 42 / 43 / 41+2 B / 42+1 B / 42+2 B$ & q \\
\hline 14 & $/ 41 / 42 / 40+2 \mathrm{~B} / 41+1 \mathrm{~B} / 41+2 \mathrm{~B} / 41+3 \mathrm{~B} / 42+1 \mathrm{~B} / 42+2 \mathrm{~B} / 42+3 \mathrm{~B} / 44+1 \mathrm{~B}$ & 우 \\
\hline 15 & 42 & q \\
\hline 16 & 42 & q \\
\hline 17 & $41+1 \mathrm{~B} / 42+1 \mathrm{~B} / 42+2 \mathrm{~B}$ & 운 \\
\hline
\end{tabular}


$\mathrm{C}$ banding method (CBG technique) applied to metaphases of the interspecies hybrids (Figure 1) showed that B chromosomes do not have any bands, suggesting the absence of heterochromatin, while the chromosome $\mathrm{Y}$ had a strong dark band which is characteristic of the red fox species. In ten autosomal chromosomes of the hybrids clear heterochromatin blocks were observed, which are characteristic of short chromosomes of the arctic fox.

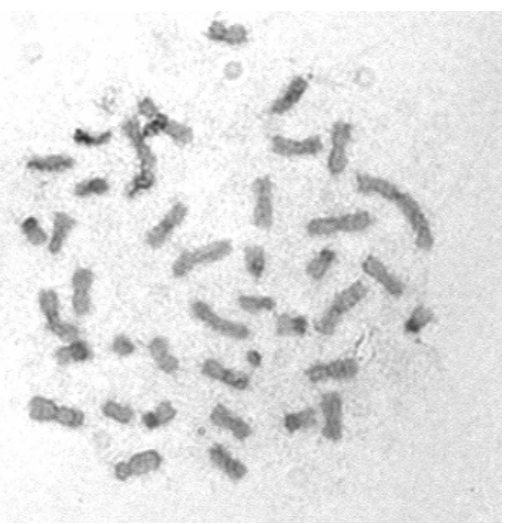

Figure 1. A metaphase plate of an interspecies hybrid (Alopex-Vulpes) (42, XY $+1 \mathrm{~B})$ stained with CBG technique

With the use of FISH technique the number of B and sex chromosomes of the interspecies hybrids (Alopex-Vulpes) was evaluated (Figure 2). On the X chromosomes, we observed a band formed after hybridization of the probe complementary to the chromosome $\mathrm{Y}$ which enabled the identification of pseudoautosomal regions.

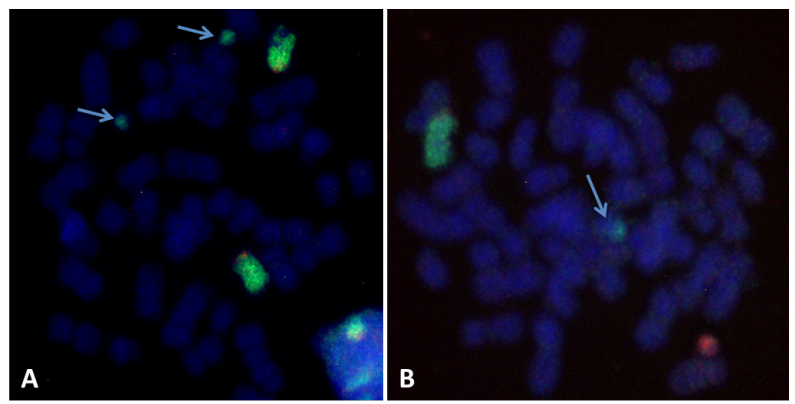

Figure 2. Metaphase plates of the interspecies hybrids (Alopex-Vulpes). FISH technique. Chromosomes $\mathrm{X}$ and $\mathrm{B}$ were visualized with a green color (FITC) and the $\mathrm{Y}$ chromosome was shown in red (Cy3). $\mathrm{B}$ chromosomes are marked with arrows. The picture A shows a metaphase of a female with two B chromosomes, and the picture B a metaphase of a male with one B chromosome

AgNOR method allowed for the visualization of active nucleolus organizer regions. For each individual a minimum of 50 metaphases were analyzed. In the arctic fox a greater number of active nucleolus organizer regions compared to the other species was observed. In the metaphases of the interspecies hybrids the most common number of NOR was indirect in relation to the parental species (Table 2). 
Table 2. The number of observed (NOO) and the most common number (MCN) of active nucleolus organizer (NOR) regions (after differential staining with AgNOR)

\begin{tabular}{l|c|c}
\hline \multicolumn{1}{c|}{ Species } & NOO & MCN \\
\hline Red fox (Vulpes vulpes) & $1-8$ & $4-5$ \\
Interspecies hybrids (Alopex-Vulpes) & $2-8$ & $5-8$ \\
Arctic fox (Alopex lagopus) & $2-9$ & $7-8$ \\
\hline
\end{tabular}

With the use of PRINS technique with specific primers for the mammalian $18 \mathrm{~S}$ ribosomal RNA gene, nucleolus organizer regions located on 12 chromosomes of the interspecies hybrid were revealed (Figure 3). The resulting hybridization signals differed in intensity and size. The presence of single copies of rDNA in the centromeric regions of few metacentric chromosomes was also observed.

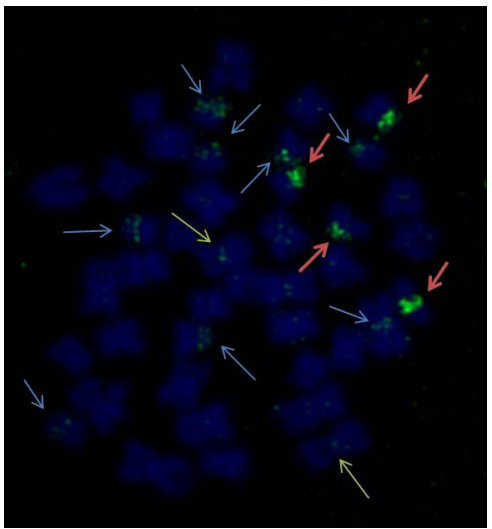

Figure 3. Metaphase plate of an interspecies hybrid (Alopex-Vulpes). PRINS technique. Red arrows indicate four large, strong fluorescent signals. Blue arrows indicate eight weaker signals. Yellow arrows indicate examples of single copies of rDNA
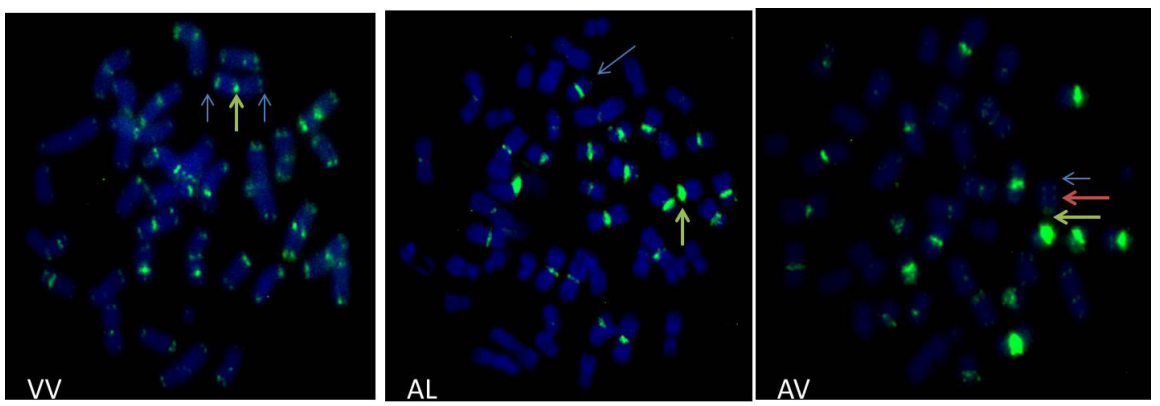

Figure 4. Metaphase plates of a red fox (VV), an arctic fox (AL) and an interspecies hybrid (AV). PRINS technique. The figure shows telomeric sequences (green - FITC). Blue arrows indicate sequences at the ends of chromosomes, yellow arrows sequences in the centromeric regions and the red arrow indicates the intrachromosomal sequence

With the use of PRINS technique telomeric sequences were revealed (Figure 4). The red fox chromosomes had telomeric sequences at the ends of chromosomes and 
in the centromeric regions. In the arctic fox, strong fluorescent signals were observed for centromeric regions and for some short chromosome arms rich in heterochromatin. Only in some chromosomes signals were present at the ends of chromosomes. Eighteen chromosomes without the fluorescent signal were found. The chromosomes of the interspecies hybrids showed signals at the ends as well as in the centromeres and short chromosome arms rich in heterochromatin. Telomeric sequences for hybrids were identified within the chromosomes.

\section{Discussion}

The crossbred of the red fox, which is characterized by a variable number of B chromosomes, and the arctic fox, having a variable number of set A chromosomes, results in the hybrids with different numbers of set $\mathrm{A}$ and $\mathrm{B}$ chromosomes, which on average is $2 \mathrm{n}=42+\mathrm{B}$ (Cole and Shackelford, 1947; Mäkinen and Gustavsson, 1982).

To date, chromosome analysis of these hybrids has been based only on the basic staining and a small number of individuals (Wipf and Shackelford, 1949; Mäkinen and Gustavsson, 1982; Świtoński et al., 1987; Gustavsson et al., 1988), whereas molecular cytogenetic techniques used in this study allowed for a broader analysis of the hybrid karyotypes.

In the case of the arctic and red fox karyotypes, chromosome numbers observed in the study were consistent with the literature $(2 n=49$ or 50 and $2 n=34$, B $1-4$, respectively). In the interspecies hybrids, the number of set A chromosomes ranged from 35 to 44 and $\mathrm{B}$ chromosome from 0 to 4 . Interestingly, not only inter-individual but also intra-individual differences in the number of chromosomes were observed, which may indicate mitotic and meiotic disorders that happened during the stage of early embryonic development leading to mosaicism. Only six individuals showed a fixed number of set A chromosomes and five hybrids did not inherit B chromosomes at all. A modal chromosome number of set A chromosomes was set to $2 \mathrm{n}=40,2 \mathrm{n}=41,2 \mathrm{n}=42$, which is consistent with the results of Mäkinen and Gustavsson (1982). In one individual, in several metaphases, 35 chromosomes were found. This can be expected to be a result of a methodological error which caused the dispersion of the metaphase plates. Such high variability of the hybrid karyotypes is a result of the variation of two parental karyotypes. As expected, the number of A chromosomes is dependent on the presence or absence of the centric fusion inherited from the arctic fox (as a parent), while the number of B chromosomes comes from the red fox. In addition, other translocations can arise due to the homology of chromosomes of artic and red foxes (Mäkinen and Gustavsson, 1982). While disturbances in divisions that occurred during the embryonic development of the hybrids could have led to ontogenetic variation within the number of chromosomes (mosaicism). The results confirm previous studies showing that B chromosomes may be inherited and that their number differs in various populations and between individuals (Świtoński et al., 1987; Green, 1988; Shi et al., 1988; Silva and Yonenaga-Yasuda, 1998) . 
The red fox supernumerary chromosomes are similar in size and morphology to the $\mathrm{Y}$ heterosome. That is why it is difficult to distinguish them when staining with standard Giemsa reagent. Therefore, the sex of the hybrids was determined by FISH method using painting probes specific to the dog heterosomes. As a result, we obtained clear signals on all chromosomes of interest. It indicates substantial interspecies conservation of the X and Y chromosomes (Bugno-Poniewierska et al., 2012). In addition, the signals obtained for the red fox on $\mathrm{B}$ chromosomes indicate the homology of these chromosomes whereas the lack of hybridization with set A chromosomes suggests that they possess no homology with the chromosomes of this set.

Moreover, $\mathrm{C}$ band staining method enables distinguishing $\mathrm{B}$ chromosomes from the $\mathrm{Y}$ heterosome. Images obtained for individual hybrids did not allow for a precise determination of their sex, but it was shown that in the studied individuals B chromosomes revealed no $\mathrm{C}$ bands, which indicate the presence of constitutive heterochromatin. However, the band was observed on the Y chromosome, which is consistent with the result obtained for the red fox (Świtoński, 1988). Furthermore, the application of CBG technique showed varying amounts of heterochromatin - small areas for the red fox compared to the large amounts for the arctic fox. In the case of the hybrids, heterochromatin blocks were observed on the short arms of small metacentric chromosomes, which is characteristic for short chromosomes of the arctic fox and is consistent with the results obtained by Mäkinen and Gustavsson (1982).

Cytogenetic studies aimed to determine the number and location of nucleolus organizer regions have shown that they are characteristic for each species. In the case of AgNOR staining a variation in number and size of silver deposits was observed. This variability is observed on the chromosomal, intercellular, interindividual and between population levels (Mellink et al., 1994; Pieńkowska and Zagalska, 2001; Słota et al., 2007). As part of these studies, we estimated the number of active nucleolus organizer regions for each of the species as well as the most common number of NORs. Regarding a general number of NORs, it was determined to be virtually identical for these species, but differences were observed in the most common number of NORs, which was the smallest for the red fox and the largest for the arctic fox; while a hybrid of the two species was a resultant of both parental species.

Although the polymorphism of silver deposits can be considered a fairly common phenomenon, the number of manuscripts describing this in detail is not too high. Frequent absence of well-documented work on the polymorphism in some species (cattle, goats, sheep) should be considered as the effect of difficulties in identifying chromosomes with nucleolus organizer regions in distal regions of long arms. In addition, the variability of the number and size of silver deposits observed after silver staining of nucleolus organizer regions appears to have a more complex etiology. Although it is possible to adopt the general assumption that the transcriptional activity of nucleolus organizer regions (expressed with parameters of silver deposits) is correlated with the number of copies of rRNA genes in the region, it is not always true that regions characterized by a large number of copies of rRNA genes exhibit a sufficiently large transcriptional activity (de Capoa et al., 1991; Mellink et al., 1994; Guillen et al., 2004). Studies on repression of transcriptional activity of nucleolus organizer regions suggest that there are at least three mechanisms causing 
inactivation of these regions. These are: the elimination of rDNA, DNA methylation and gene silencing by the effect of the position within the heterochromatin or telomeres (Wnuk et al., 2006). The results of fluorescently visualized rDNA sequences allowed distinguishing four nucleolus organizer regions with strong fluorescent signals and six regions with weak fluorescent signals. Furthermore, single rDNA signals in the centromeric regions of several chromosomes of the interspecies hybrids were observed. These observations support the possibility of inactivation of nucleolus organizer regions by the above-mentioned mechanisms.

In order to visualize the telomeric areas of the examined species PRINS technique with primers specific to telomeric sequences was used. The results of this study show the presence of telomeric sequences in the red fox, the arctic fox and in their hybrids at both ends and inside the chromosomes. The presence of telomeric sequences within the chromosomes (ITS - Interstital Telomeric Sequences) may be due to multiple rearrangements that have occurred during speciation in the Canidae family, such as tandem or centric fusions (Robertsonian translocation) and other fusions or inversions. ITS can also arise during the repair of double-strand breaks (DSB) (Ruiz-Herrera et al., 2008). Some studies suggest that the presence of ITS in the region of constitutive heterochromatin is not due to chromosomal rearrangements, but they are part of the satellite DNA (Garagna et al., 1997; Pagnozzi et al., 2000; Multani et al., 2001; Pagnozzi et al., 2002).

The results of this study showed that chromosomal markers of the interspecies hybrids are inherited from the parent species and are a result of combination of their two genomes. However, intraindividual differences are also observed which may result from chromosomal aberrations during embryonic development. This may lead to the formation of different cell lines with different karyotypes (mosaicism). The applied methods of molecular cytogenetics allowed broadening the knowledge about the karyotype of the interspecies hybrids.

\section{References}

B a sh ev a E.A., Torga sh ev a A.A., S a k a e v a G.R., B i d a u C., B or o d in P.M. (2010). A- and B-chromosome pairing and recombination in male meiosis of the silver fox (Vulpes vulpes L., 1758, Carnivora, Canidae). Chromosome Res., 18: 689-696.

B e li a e v D.K., Vol obuev V.T., R a dzhabli S.I., Trut L.N. (1974). Supernumerary chromosome polymorphism and mosaicism in silver foxes. Genetika, 10: 58-67.

Bugno-Poniewierska M., Sojecka A., Pawlina K., Jakubczak A., Jeżewska- W it k o w s k a G. (2012). Comparative cytogenetic analysis of sex chromosomes in several Canidae species using Zoo-FISH. Folia Biol., 60: 11-16.

Cole L.J., Shackelford R.M. (1947). Fox hybrids. Transactions of the Wisconsin Academy of Sciences, Arts and Letters, 38: 315-332.

de Capoa A., A le ixandre C., Felli M.P., Ravenna L., Costantino M.A., Giancotti P., Vicenti O., Pogges i I., Grappelli C., Miller D.A. (1991). Inheritance of ribosomal gene activity and level of DNA methylation of individual gene clusters in a three generation family. Hum. Genet., 88: 146-152.

Garagna S., Ronchett i E., Mas cherett i S., Crovella S., Formenti D., Rumpler Y., R o m a n i M.G.M. (1997). Non-telomeric chromosome localization of (TTAGGG)n repeats in the genus Eulemur. Chromosome Res., 5: 587-591. 
Gre e n D.M. (1988). Cytogenetics of the endemic New Zealand frog, Leiopelma hochstetteri: extraordinary supernumerary chromosome variation and a unique sex-chromosome system. Chromosoma, 97: 55-70.

Guill e n A.K., Hirai Y., Tan o u e T., H ir a i H. (2004). Transcriptional repression mechanisms of nucleolus organizer regions (NORs) in humans and chimpanzees. Chromosome Res., 12: 225-237.

Gust a v s s o n I., S und t C.O. (1965). Chromosome complex of the family Canidae. Hereditas, 54: $249-254$.

Gustavs s o n I., S und t C.O. (1967). Chromosome elimination in the evolution of the silver fox. J. Hered., 58: 75-78.

Gustavs on I., Św it oński M., Larsoson K., Ploen L. (1988). Synaptonemal complex analysis of spermatocytes in hybrids of silver fox and blue fox. J. Hered., 79: 338-344.

H ow e 11 W.M., B l a c k D.A. (1980). Controlled silver-staining of nucleolus organizer regions with a protective colloidal developer: a 1-step method. Experientia, 36: 1014-1015.

Mäk in en A. (1985 a). The standard karyotype of the blue fox (Alopex lagopus L.). Committee for the standard karyotype of Alopex lagopus L. Hereditas, 103: 33-38.

Mäk in e n A. (1985 b). The standard karyotype of the silver fox (Vulpes fulvus Desm.). Committee for the standard karyotype of Vulpes fulvus Desm. Hereditas, 103: 171-176.

Mäk in e n A., Gu s t a v s s o n I. (1982). A comparative chromosome banding study in the silver fox, the blue fox, and their hybrids. Hereditas, 97: 289-297.

Mellink C.H., B o s m a A.A., D e H a a n N.A. (1994). Variation in size of Ag-NORs and fluorescent rDNA in situ hybridization signals in six breeds of domestic pig. Hereditas, 120: 141-149.

Multan i A.S., O zen M., F urlong C.L., Zh a o Y.J., H s u T.C., P a tha k S. (2001). Heterochromatin and interstitial telomeric DNA homology. Chromosoma, 110: 214-220.

Pagnozzi J.M., Silva M.J.J, Yonenaga-Yas suda Y. (2000). Intraspecific variation in the distribution of interstitial telomeric (TTAGGG)n sequences in Micoureus demerarae (Marsupialia: Didelphidae). Chromosome Res., 8: 585-591.

P a g n o z zi J.M., D i t c h fi e ld A.D., Yon en a ga - Ya s s u d a Y. (2002). Mapping the distribution of the interstitial telomeric (TTAGGG)n sequences in eight species of Brazilian marsupials (Didelphidae) by FISH and the correlation with constitutive heterochromatin. Do ITS represent evidence for fusion events in American marsupials? Cytogenet. Genome Res., 98: 278-284.

P i eńk ow s k a A., Z a g a ls k a M. (2001). Comparison of the Nuclear Organiser Region activity in four taxa of the family Canidae. J. Appl. Genet., 42: 493-501.

Ruiz-Herrera A., Nergadze S.G., Santagostino M., Giulot to E. (2008). Telomeric repeats far from the ends: mechanisms of origin and role in evolution. Cytogenet. Genome Res., 122: 219-228.

S h i L., Tang L., M a K., M a C. (1988). Synaptonemal complex formation among supernumerary B chromosomes: an electron microscopic study on spermatocytes of Chinese raccoon dogs. Chromosoma, 97: 178-183.

S h o r t R.V. (1997). An introduction to mammalian interspecific hybrids. J. Hered., 88: 355-357.

S ilva M.J., Yonen a g - Yas s u da Y. (1998). Heterogeneity and meiotic behavior of B and sex chromosomes, banding patterns and localization of (TTAGGG)n sequences by fluorescence in situ hybridization in neotropical water rat Nectomys (Rodienta, Muridae). Chromosome Res., 8: 305-311.

Słota E., Wnuk M., Bugno M., Pieńkowska-Schelling A., Schelling C., Brat u s A., K o t y la k Z. (2007). The mechanisms determining the nucleolar-organizing regions inactivation of domestic horse chromosomes. J. Anim. Breed. Genet., 124: 163-171.

Ś w i t oń s ki M. (1981). Robertsonian translocation in the blue fox (Alopex lagopus) and its effect on the fertility. Genet. Polonica, 22: 463-474.

Św i toń s ki M. (1984). Preliminary investigation on inheritance of the B-chromosomes in silver fox (Vulpes vulpes). Proc. 6th Eur. Colloq. Cytogenet. Domest. Anim., pp. 303-310.

Ś w i toń s k i M. (1985). Study on the inheritance of the centric fusion in the blue fox (Alopex lagopus). Genetica, 68: 65-68.

Ś witoń s ki M. (1988). B chromosomes in the fox (Vulpes vulpes), their nature, distribution, inheritance and effects. Ann. Agr. Univ. Pozn., p. 174.

Św i toń ski M., Gustavs s o n I., Hojer K., P loen L. (1987). Synaptonemal complex analysis 
of the B-chromosomes in spermatocytes of the silver fox (Vulpes fulvus). Cytogenet. Cell Genet., 45: 84-92.

Ś w i toń s k i M., R og a ls k a - N i żn i k N., S z c z e r b a l I., B a e r M. (2003). Chromosome polymorphism and karyotype evolution of four canids: the dog, red fox, arctic fox and raccoon dog. Caryologia, 56: 375-385.

Wipf L., Sha c kelford R.M. (1949). Chromosomes of a fox hybrid (Alopex-Vulpes). Proc. Natl. Acad. Sci., 35: 468-472.

W n u k M., S ł o t a E., K o t y l a k Z., B u g n o M. (2006). Factors responsible for modulation of ribosomal RNA transcription. Folia Biol., 54: 1-8.

Received: 25 VII 2014

Accepted: 12 IX 2014 